10 years ESJ

Special edition

\title{
Intercultural Aspects of Specialized Translation. The Language of Traditional Chinese Medicine in a Globalized Context
}

\author{
Barbara Cappuzzo \\ University of Palermo, Italy
}

\section{Doi:10.19044/esj.2022.v18n5p25}

Submitted: 11 November 2021

Accepted: 18 January 2022

Published: 21 February 2022
Copyright 2022 Author(s)

Under Creative Commons BY-NC-ND

4.0 OPEN ACCESS

Cite As:

Cappuzzo B. (2022). Intercultural Aspects of Specialized Translation. The Language of Traditional Chinese Medicine in a Globalized Context. European Scientific Journal, ESJ, 18 (5), 25. https://doi.org/10.19044/esj.2022.v18n5p25

\section{Abstract}

Traditional Chinese Medicine (TCM) has a very ancient history and its importance for human health has long been acknowledged worldwide. The significant role that TCM plays in the global healthcare system has been officially recognized by the World Health Organization (WHO), which devoted a chapter to TCM in the 2019 latest version of the International Classification of Diseases and Related Health Problems (ICD-11). TCM, alone or in combination with Western Medicine (WM), has been recommended for various health conditions (Aung et al., 2013; Cai et al., 2015; Zhang et al., 2015) - including mild COVID-19-related symptoms (Zhao et al. 2021). As a result of globalization, which has fostered the spread of TCM in the Western world, and the prominent role of English as the language of international communication, there has been an ever-increasing need for translation and higher levels of accuracy and standardization in the English terminology related to Chinese medical concepts. This issue has raised several debates over time among linguists, translators, and physicians as to the approach to be adopted for the English translation of Chinese medical terms. If on the one hand, a source-oriented strategy tends to preserve the original meaning and to convey concepts as closely as possible to the original language, on the other hand, a target-oriented method attempts to render those concepts more accessible to the target language, albeit the integrity of their true meaning is likely to be undermined. This paper intends to highlight the 
major questions that the translation of culture-bound terms poses when different cultures are involved. In this respect, two important works were analyzed and compared, namely A Practical Dictionary of Chinese Medicine (1998), and the International Standard Chinese-English Basic Nomenclature of Chinese Medicine (2008). Both works testify to the difficulty of carrying out advanced projects of standardization of Chinese-English nomenclature of medical terms in the joint effort to create a common reference language.

Keywords: Traditional Chinese Medicine, English translation, sourceoriented translation, target-oriented translation, terminology standardization

\section{Introduction}

Traditional Chinese Medicine (TCM) is a very ancient medical system that dates back to more than five thousand years ago. It encompasses a wide range of therapies, including acupuncture, acupressure, moxibustion, herbal medicine, massage, and practices such as Tai Chi and Qigong. Acupuncture, in particular, has been recognized by the World Health Organization (WHO) for the treatment of various illnesses and disorders, as a result of extensive studies on its effectiveness (Witt et al., 2006; Yuan et al., 2008; Manheimer et al., 2010; Hinman et al., 2014; Vickers \& Linde, 2014; Linde et al., 2016). In the twentieth century, China developed three healthcare systems, i.e. TCM, Western Medicine (WM), and Integrated Medicine (IM) (Keji \& Hao, 2003). The latter is increasingly fostered as a comprehensive global approach to the health care in the Western world, in that TCM and conventional WM are considered as complementing each other, with TCM being widely appreciated for its holistic approach and lack of adverse effects.

TCM has been growing very fast following the rapid economic expansion of China since the late 90s. As an effect of globalization, it has spread to 183 countries and regions worldwide. Moreover, 103 countries have approved the practices of acupuncture and moxibustion, 29 have enacted statutes on TCM, and 18 have included acupuncture and moxibustion in their insurance provisions (Xu \& Xia, 2019). Some scholars (Fears et al., 2019; Zhou, 2019; You, 2020) have highlighted the fact that the efficacy and safety of TCM and its practice have not yet been validated by solid scientific studies. However, a chapter on TCM has been included in the latest version of the WHO International Classification of Diseases and Related Health Problems (ICD-11, 2019) for the first time. ICD-11 was presented at the World Health Assembly in May 2019 for adoption by Member States and will come to effect on January 1, 2022. It includes TCM diagnostic elements such as Qi

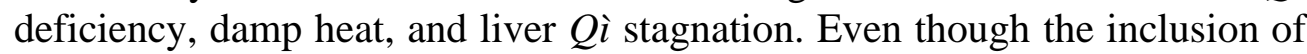
TCM in ICD-11 does not represent a judgment on its validity and efficacy, it is a very important milestone, as the importance of TCM within the global 
healthcare system has been sanctioned, and advancements and developments in services, education, research, and regulation will presumably be fostered. In this respect, Lam et al. (2019: 373) state:

including TCM in ICD-11 [...] enables the statistical data to cover information beyond Western medicine, thus reflecting the true situation of healthcare systems among member states of the WHO. [...]. In addition, this initiative contributes to the progressive reform of the world healthcare system, from the global extension of TCM to the integration of TCM with multiple Western medicine-based disciplines.

From a linguistic viewpoint, globalization and the spread of TCM worldwide have led to an increased need for translation. This, in turn, has determined a search for standardization of the English terminology of Chinese medicine, also as a consequence of confusion caused by heterogeneity of the terms used in texts published in China and other countries. Hard work carried out for many years by linguists, medical professionals, sinologists, and scholars of Chinese medicine and culture resulted in the creation of the World Federation of Chinese Medicine Societies' (WFCMS) International Standard Chinese-English Basic Nomenclature of Chinese Medicine (2008), an effort that involved more than 200 specialists from 68 countries and 70 academic institutions of Chinese medicine.

The aim of this work is to discuss some aspects of the translation of TCM terms into English and the major issues that culture-bound translation shows when very different conceptual systems are involved. The study will also try to highlight, on the one hand, the need for standardization of the English terminology of traditional Chinese medical concepts, and, on the other hand, the difficulty of obtaining definitive results on both the semantic and the methodological levels.

Before going into the core of the work, it was deemed appropriate to discuss some important aspects of the theoretical foundations that underpin TCM, to provide the cultural framework that is necessary to better understand the debate on the English translation of Chinese medical terms.

\section{TCM basic concepts}

TCM is founded on a holistic approach to the human body and the universe, as described in Taoism (or Daoism). More specifically, a close relationship exists between the body and its natural environment, in that the laws that regulate the human body are the same as those governing the universe, and this results in an "integrity between human and cosmos" ( $\mathrm{Lu}$ et al. 2004).

The basic tenets of TCM can be found in Wiseman and Ellis' Fundamentals of Chinese Medicine (1996), a translation of Zhong Yi Xue Ji. As the authors explain in the preface to the book, the importance of the work 
lies not only in the fact that it is one of the few direct translations of primary Chinese sources but also in its being the result of hard cooperation "meant to achieve the highest precision in the rendering of Chinese medical concepts" (Wiseman \& Ellis, 1996: ix). The first chapter of Fundamentals of Chinese Medicine deals with the Yin-Yang Theory and the Five Phases Theory, two basic concepts with which the ancient Chinese explained all phenomena regarding the universe and human beings. "All phenomena in the universe may be ascribed to yin and yang. Each phenomenon possesses both a yin and a yang aspect" (Wiseman \& Ellis, 1996: 1). The Yin-Yang Theory explains the way the universe is regulated, that is in groups of opposites (e.g., sun/moon, night/day, male/female). Yin and Yang are interdependent, they counterbalance each other, and each Yin and Yang phenomenon has Yin and Yang aspects that can be divided in the same way ad infinitum. The concept of the interdependence of Yin and Yang also concerns medicine, explaining all physiological, pathological, and therapeutic aspects and phenomena. With specific reference to diseases, since Yin and Yang cannot exist without each other, a deficit of one inevitably leads to a surfeit of the other, and the result is a disease. In other words, if one component predominates over the other, a condition of Yin-Yang imbalance is generated, and health is in some way compromised.

The Five Phases Theory is based upon the concept according to which all phenomena are the result of the interaction of five elements, namely Wood, Fire, Earth, Metal, and Water. The interaction of the five elements gives rise to four cycles, which explain the physiological activities and pathological conditions in the human body. In TCM, organs correspond to natural phenomena and are classified based on five phases. Hence, the liver is associated with wood, the heart with fire, the spleen with earth, the lung with metal, and the kidney with water. The Five Phases Theory plays a very important role in medicine, influencing physiology, diseases, diagnosis, treatment, and pharmacology.

Another fundamental concept of TCM is that of $Q i$ (which will also be discussed in section 3), whose simplified primary logograph is 气. The most common character for $Q i$ is 氣. It is made up of two pictograms, 气 representing air, and 米 representing rice or grain. Therefore, it can be interpreted as a sort of vapor coming out of rice (or grain). The image displayed by the ideogram 氣 conveys the concept of something which is, at the same time, both material and immaterial. $Q i$ is the most discussed term when dealing with the translation of Chinese medical terms into English (Cappuzzo, 2009). Several attempts have been made to offer an adequate English equivalent for this term. Lloyd and Zhao (2018: 138) define Qì as 
"breath/energy", and Drewe (2011: 35) offers a thorough list of equivalents for $Q i$ and an explanation of its meaning:

Literally, qì translates as any of the following: 'gas / air / steam / vapour / smell / weather / vital breath / to anger / to get angry / to be enraged'. [...] Usually in the West, the word is used to mean 'energy' or 'vital energy'. Qì is the vital force without which we would be unable to exist. In the West, we would say that someone with a strong constitution has strong qì; it is the power that fuels our bodies and minds, and keeps us healthy.

The duality of the term and the different meanings it encompasses make it a hard task to find an Italian equivalent. In this respect Maciocia (2015: 44) states:

It is very difficult to translate the word Qi and many different translations have been proposed, none of which approximates the essence of Qi exactly. It has variously been translated as 'energy', 'material force', matter, ether, matter-energy, vital force, life force, vital power, moving power. The reason it is so difficult to translate the word Qi lies precisely in its versatile nature whereby Qi can assume different manifestations and be different things in different situations. [...]. Because of the difficulty in finding an appropriate translation for the term Qi, I have chosen to leave it untranslated, as I do for 'Yin' and 'Yang'."'

In ancient Chinese philosophy, $Q i$ is believed to be a subtle substance (Wiseman, 2001), the entity that explains all phenomena in the universe. Qi flows in the body through channels made up of linear groups of points situated along the skin and called meridians. The activity of the different forms of $Q i$ (e.g. original $Q i$, construction $Q \grave{i}$, defense $Q i)$ support the physiological and pathological conditions.

What has been said for $Q i$ in Chinese philosophy also applies to Chinese medicine and the human body. Chinese medicine emphasizes the strong relationship between human beings and nature and takes it into account when dealing with diseases, etiology, and treatment. In the human body, there are different forms of $Q \grave{i}$, going from the most immaterial and rarefied to the very tangible and physical ones.

Those mentioned so far are only some of the innumerable examples of concepts that belong to TCM. However, they can give an idea of the complex issue that arises when dealing with the translation of Chinese terms into English and of the implications that different translation approaches may have.

\section{Methods}

The analysis of the translation into English of Chinese terms took into account the two major and most authoritative works on the topic, A Practical Dictionary of Chinese Medicine (1998), by Wiseman and Ye, which is a book halfway between a manual and a dictionary, and the previously mentioned 
World Federation of Chinese Medicine Societies' (WFCMS) International Standard Chinese-English Basic Nomenclature of Chinese Medicine (2008) ${ }^{1}$. Both works serve a normative function and make an important contribution to translation studies, as they also include discussion of the main issues deriving from the westward transmission of Chinese medical concepts. A Practical Dictionary is, to date, the most authoritative encyclopedic work in the English literature about Chinese medicine, and has largely contributed to the understanding and transmission of TCM concepts worldwide. It includes nearly 6,000 medical terms and is an invaluable source of useful clinical information. Acupuncture is given much space and medicinal treatments are included for most diseases. The International Standard is the reference standard nomenclature in 174 WFCMS member societies in 55 regions and countries. It includes 6526 terms and the most widely accepted and updated English translations.

In this study, A Practical Dictionary and the International Standard were compared to investigate some crucial terms, with particular attention to $Q i$, which refers to one of the most discussed concepts in the debate on the most appropriate Western equivalents for TCM terminology.

The analysis also took into consideration the concepts of feng huo yan and shi du chang, which are two significant examples of the implications that completely different translation approaches can have in the westernization of TCM concepts.

The analysis highlighted the importance given, in A Practical Dictionary, to the literal translation, considered as the only valid approach to preserving the meaning of the original Chinese concepts. The effort, shown in the International Standard, to avoid improper use of modern medical equivalents that do not respect the original Chinese meanings was also discussed. For this purpose, the terms shen zhu shiu and xio ke were analyzed. Finally, the investigation examined the International Standard dual translation as for denominations of diseases mostly concerning ophthalmology.

\section{Results and Discussion}

The first investigated term is the above-mentioned $Q i$. In English, $Q i$ is almost always used as a loanword as it entered the language through long usage. The International Standard registers $Q i$ as untranslated both when occurring as an isolated entry and when associated with another term to form compounds (e.g. Qì transformation', 'Qì movement', etc.). This is because $Q i$ - as well as Yin and Yang - entered the English language a long ago and

${ }^{1}$ Henceforth A Practical Dictionary of Chinese Medicine and International Standard Chinese-English Basic Nomenclature of Chinese Medicine will be referred to, respectively, as A Practical Dictionary and International Standard for convenience. 
therefore it has become quite 'familiar' in the Western world. A Practical Dictionary provides the reader with the variety of meanings that $Q i$ encompasses. More precisely, the dictionary registers twelve equivalents for $Q i$, which are all worth mentioning to have an idea of the polysemous nature of the term, thus also shedding light on the complexity of many other TCM fundamental concepts revolving around $Q i$ :

1. Air, gas, vapor, flatus. 2. Smell. 3. Aura. 4. Environmental forces. 5. Nature (e.g., the Four QI). 6. Anything of a particular nature (e.g., Yin QI). 7. Breath (e.g., SHORTAGE OF QI AND QI SHORTAGE). 8. Any of various dynamic phenomena of the body (e.g. SOURCE QI, CONSTRUCTION QI [...]. 8a. Activity: Qi is highly active; human growth and development, as well as physiological activity and metabolism, are manifestations of the activity of qi. 8b. Warming: The temperature of the human body and the ability of the organs and tissues to perform their functional activities are dependent on the warming action of qi. 8c. Defense: Qi is the outer defense of the body, and prevents evils from entering. 8d. Transformation: Production of blood and fluids, the distribution of fluids, and the conversion of fluids into sweat and urine are all the results of qi transformation. Implicit in this notion is the movement, or bearing, of qi. Qi bears upward, downward, inward and outward, carrying blood and fluids with it. Combinations of these four movements explain all movement in the body. [...] 8e. Containment: Under normal circumstances, spillage of blood from the vessels is prevented by the containing function of qi. Hence, it is said, "Qi contains the blood." [...] 9. Strength. 10. Anger. 11. Disease (e.g. LEG QI, [...]). 12. An abbreviation for diseases of qi (qi vacuity, qi stagnation), [...].

The definitions given by Wiseman and Ye (1998) testify to the complexity of the issue concerning the meaning of $Q i$ and the difficulty of finding a single equivalent that can thoroughly encompass all the concepts it refers to.

Maciocia (2015: 44) emphasizes the fact that most sinologists generally agree that $Q i$ is conceived as 'matter', although not 'matter' in a restricted materialistic connotation, as $Q i$ can assume non-material forms. The scholar adds that in Chinese philosophy there is a specific term to indicate

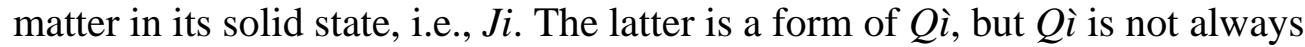
$J i$, because, as already mentioned, $Q i$ may manifest itself in both material and immaterial forms.

The question of translatability/untranslatability of Chinese medical terms into English mainly depends on the conceptualization of terms according to Western culture. In this respect, in the preface to A Practical Dictionary, Wiseman and Ye (1998: viii) specify that they "have avoided translations of terms that might encourage readers to attach any modern Western ideas to them." The scholars report the example of feng huo yan, 
which both they and the International Standard render as "wind-fire eye". However, feng huo yan is commonly translated as "acute conjunctivitis". Wiseman and Ye (1998: viii) state that the literal translation of this term preserves for the Western reader the Chinese medical etiology of the disease and avoids the impression that Chinese medicine has either the anatomical concept of the conjunctiva or the pathological concept of inflammation ('itis'), as well as obviating the need to establish whether feng huo yan corresponds in all cases to the Western medical notion of conjunctivitis.

Wiseman and Ye reject the Western medical translation 'acute conjunctivitis' because even though on the level of the meaning the issue is the same, that is a condition of discomfort and redness of the eye, on the theoretical level the original term and its Western translation belong to completely different conceptual systems. Besides having neither the concept of conjunctiva nor that of inflammation ('-itis'), the causes of a red eye may be numerous, including that "of having one drink too many" (Hai 2014: 54). In TCM medical culture, alcohol stokes fire in the liver and mobilizes wind upwards inflaming the eyes. In Western medicine, alcohol causes liver alterations up to cirrhosis that result in yellow eyes, due to accumulation of liver pigments such as bilirubin and biliverdin. Moreover, in TCM, eye redness is not necessarily associated with an infection or with allergy as in the case of acute conjunctivitis.

In TCM, there are several examples of terms that have undergone a process of adaptation to Western culture and are based on completely different clinical interpretations. Besides feng huo yan, another example is shi du chuang, literally translated "damp toxin sore" in Wiseman and Ye's (1998) A Practical Dictionary. The latter also registers the Western English equivalent "eczema", which is the only equivalent reported by the International Standard. In Western medical culture, the term "eczema" refers to "an inflammatory condition of the skin characterized by redness, itching, and oozing vesicular lesions which become scaly, crusted, or hardened" (MerriamWebster, merriam-webster.com). By contrast, in TCM "damp toxin sore" is defined as "a sore attributed to wind, dampness, and heat lodged in the skin. [...]. Chronic forms, which are complicated by blood vacuity, are characterized by drying, thickening, and scaling of the skin" (Wiseman \& Ye, 1998: 117). As can be seen, two different descriptions are made of the same 'object' named "damp toxin sore"/ "eczema". In the former, "wind", "dampness", "heat", and "blood vacuity" are all terms referring to the pathophysiology of $Q i$, and therefore they belong to a very different conceptual system as compared to the Western one. "Dampness", for example, is not to be considered in the Western sense of concentration of water vapor in the air, but as a condition of humidity inside the body. Despite being associated with dampness in the natural environment, "dampness is a yin 
pathogen that impairs yang and causes Qì stagnation" (Block, 2008: 1). Dampness is caused by spleen $Q \grave{\imath}$ deficiency and causes liver $Q \grave{\imath}$ stagnation. Dampness starts from the lower extremities, and then settles in the lower jiao, ${ }^{2}$ from there spreading throughout the body. Patients with dampness suffer from dizziness, joint soreness and pain, and heaviness in the head, some of the many other possible symptoms (Qu, 2018).

Wiseman and Ye's (1998) position about the importance of a sourcelanguage translation approach in TCM terminology is based on the belief that full understanding of Chinese medicine and of its intimate concepts is achieved by absorption of the original meanings, which can be transferred only through literal, source-oriented translations. The scholars also appeal to the fact that in Chinese medicine the relationship between term and concept is not as clear as in WM, in that there are terms that can have different meanings and it is not always possible to infer from the context what specific meaning an author refers to. An example is xue hai, which literally means "sea of blood". Xue hai has three different meanings, namely the Thoroughfare Vessel (one of the Eight Extra Meridians), the liver, and SP-10 (an acupuncture point). The latter is the only equivalent registered in the International Standard, whereas A Practical Dictionary reports the literal translation. In this respect, Wiseman (2001) believes that a good translation is one that maintains the same degree of ambiguity as in Chinese, and this can only be attained through literal translation. In other words, although ambiguity is also conveyed to the target language, it is better to remain faithful to the original meaning rather than to risk wrong and misleading interpretations.

Now one wonders what outcomes the International Standard has reached, what inspiring principles have been followed, and what approaches have been adopted for the English translation of Chinese medical terms. In the introduction to the work, it is said that the English translations of Chinese terms fit the original meanings and this "is the most important principle among other principles" (2008: xxxiv). It is also specified that possible English translations that fall outside the original meaning have been registered as they have been accepted after long usage.

A very important step taken by the International Standard is the corrective action against improper use of modern English medical equivalent employed to translate some Chinese terms. For example, it is stated that shen zhu shiu must be translated as "kidney governing water" and not as "kidney governing water metabolism". The reason lies in the fact that in Western terms the concept of metabolism describes all chemical reactions involved in maintaining the state of the cells in living beings. In TCM, the Western

2 "The lower jiao is the area below the navel. In TCM, it contains the kidney and liver, urinary bladder, large intestine, small intestine, uterus, legs, and feet. "Because the lower jiao discharges waste from the body, it is described as the "drainage" (du)" (Qu 2018: 85). 
concept of metabolism corresponds to what must always be attributed to the action of $Q i$, and in the specific case of the kidney, to the action of jing $Q i$ (also called essential $Q i$ ), which is stored in the kidneys and is responsible for the regulation of processes like growth, development, digestion, and reproduction. What in WM is called water metabolism regarding the kidney is to be seen, in TCM, as a sort of 'distillation', that is the regulation of the distribution of fluids in the body. Wiseman and Ye (1998: 328) explain that "through the spleen's movement and transformation, and the lung's regulation of the waterways, fluids are distributed throughout the body, and waste water is carried down to the bladder before being discharged." This is the $Q i$ transformation of kidney, which plays a fundamental role in the state of wellbeing of the human body (Dong, et al. 2017: 318). What has just been said explains the reason for not including the term "metabolism" in the translation of shen zhu shiu, as it would entail a deviation from the correct interpretation of the authentic meaning. Another corrective action made by the International Standard concerns the English translation of names of diseases when these show more than one equivalent. It is the case of xio ke, whose literal equivalent "consumptive thirst" has been provided, and none of the most commonly used 'equivalents', that is "diabetes mellitus", "diabetes insipidus", and "psychogenic polydipsia" were included (Duan et al., 2021). Given that three different pathologies are dealt with, the literal translation was preferred to denominations of diseases that could cause problems of ambiguity, especially "diabetes mellitus" and "diabetes insipidus", of which excessive thirst represents one of the symptoms, but does not identify the disease itself. Most importantly, the concepts of diabetes mellitus, diabetes insipidus, and psychogenic polydipsia are very far from the meaning that "consumptive thirst" assumes, on the pathophysiology level, in TCM. Here, the term refers to the consequence of several so-called "patterns of disharmony" (Dong et al., 2017; Huang et al., 2020). A pattern is when the harmony of the body system is disrupted, leading to symptoms or signs that something is wrong ("pattern" is similar to, but not quite the same as, "disease"). One characteristic symptom of what in WM is termed "diabetes mellitus" is excessive thirst, which in TCM is described as kidney Yin deficiency along with lung Yin deficiency and internal heat that consumes fluids, thus causing thirst (Covington, 2001). As happens for all health problems, in this case, too, what is dealt with is a condition ultimately attributable to an imbalance of $Q i$.

Besides terms where only the literal translation is provided, the International Standard also displays dual translation, i.e. the use of several terms whose word-for-word translation is accompanied by Western equivalents. These terms mostly concern ophthalmic diseases, as in TCM the eye plays a crucial role. It is referred to as the shen, that is the vital pilot, and the diseases affecting the eye are considered to have a direct connection with 
psychological conditions. Some examples of Chinese terms that show an English literal equivalent next to a Western one are "wind red sore" / "eyelid dermatitis", "red vessels crossing white eye" / "angular conjunctivitis", "malnutrition involving eye" / "keratomalacia", and "high-wind internal visual obstruction" / "pigmentary retinopathy". The above-mentioned examples also show the important role that the literal translation plays, as it immediately provides information about the pathophysiology of the diseases.

In the preface to the International Standard, it is specified that in the case of a denomination of disease having a single equivalent in WM, the latter must be reported next to the literal translation of the Chinese denomination. However, there are cases of denominations of diseases whose only equivalent is a Western one and no loan translation has been provided next to it. Two instances are yin zhen, which literally means "dormant papules" and whose only registered equivalent is "urticaria", and mian you feng, which only shows "facial seborrheic dermatitis", and no literal translation ("face wandering wind") is displayed.

\section{Conclusion}

The spread of TCM worldwide has made the standardization of the English terminology of Chinese concepts a crucial issue. Much progress has been made but much work still needs to be done in this respect. The International Standard is the result of hard work involving linguists and sinologists from all over the world. Today, this work and the enormous effort it encompasses prove even more precious in the light of the recent inclusion of TCM in the latest edition of ICD, which has sanctioned the importance of Chinese medicine within the global healthcare system. However, the International Standard also testifies to the difficulty of coming to a unique and homogeneous solution in the creation of a corresponding English terminology of TCM concepts. This is due to the fact that the translation approach to be adopted depends on several different factors, including the purpose of the translation and the target the message is addressed to. If the translation concerned, for instance, classic texts describing the original thinking of the ancients, it would be more appropriate to adopt a sourceoriented approach, as it is the only one that can preserve the integrity of the original concepts. By contrast, if translation concerned modern texts designed for educational purposes, a target-oriented approach might prove more useful. However, evaluation of any correctness/ inappropriateness of the existing Western equivalents becomes necessary. It is in this regard that Hai (2014: 57) suggests including Chinese vocabulary in the English translation of TCM concepts. In the scholar's opinion, this method would offer the twofold advantage of not taking the risk of using possible inappropriate Western 'equivalents' and avoiding shifting source-language-related ambiguities to 
loan translations. However, this approach shows the limit that loanwords, unless well rooted after long usage, may prove meaningless to the Western reader, who may need explanations.

The International Standard use of dual translation for a considerable number of terms, especially those related to disease denomination may represent an adequate suggestion in the translation of TCM texts into English. The juxtaposition of literal translations and conventional terms offers the twofold advantage of rendering TCM concepts more accessible to the Western audience and, at the same time, preserving the original meanings. Most importantly, it emphasizes the mutual differences between two extremely different medical systems, and by doing so, it contributes to a greater understanding of the meanings from more cultural perspectives.

Finally, attention should be paid to the importance of promoting Chinese language learning to better understand TCM crucial concepts and first and foremost fill the gap of the still few existing translations based on primary sources. This would help, perhaps, solve some conceptual ambiguities and pave the way to an easier process of terminology standardization.

\section{References:}

1. Aung S.K., Fay H. and R.F. Hobbs, $3^{\text {rd }}$ (2013). Traditional Chinese Medicine as a Basis for Treating Psychiatric Disorders: A Review of Theory with Illustrative Cases. Med Acupunct., 25 (6), (pp. 398-406).

2. Block E. F. (2008). TCM Dampness and Food Therapy. Acupuncture Today, 9 (4),

https://acupuncturetoday.com/mpacms/at/article.php?id=31706

3. Cai Y., Boyd D. L., Coeytaux R.R., Østbye T., Wu B. and Z. Mao (2015). Treatment of Chronic Conditions with Traditional Chinese Medicine: Findings from Traditional Chinese Medicine Hospitals in Hubei, China. The Journal of Alternative and Complementary Medicine, 21(1), (pp. 40-45).

4. Cappuzzo B. (2009). The Translation of Chinese Medical Terms into English. Linguistic Considerations on the Language of TCM. Journal of Sports Sciences and Law, 2 (3), (pp. 49-60).

5. Covington M.B. (2001). Traditional Chinese Medicine in the Treatment of Diabetes. Diabetes Spectrum, 14 (3), (pp. 154-159).

6. Dong J., Wang T., Zhao L. and X. Chen (2017). Pattern of disharmony between the heart and kidney: Theoretical basis, identification and treatment. Journal of Traditional Chinese Medical Sciences 4 (4), (pp. 317-321).

7. Drewe J. (2011). The Yang Tàiji 24-Step Short Form: A Step-by-Step Giude for all Levels. London and Philadelphia, Singing Dragon. 
8. Duan X., Wang W., Pan Q. and L. Guo (2021). Type 2 Diabetes Mellitus Intersects with Cancer Diagnosis and Development. Front. Oncol., 11, https://www.ncbi.nlm.nih.gov/pmc/articles/PMC8415500/

9. Fears R., Griffin G.E., Larhammar D., ter Meulen V. and W.M. der Meer (2019). Globalization of Traditional Chinese Medicine: what are the issues for ensuring evidence-based diagnosis and therapy?, Journal of Internal Medicine, 287 (2), (pp. 210-213).

10. Hai H. (2014). The Theory of Chinese Medicine. A Modern Interpretation. London, Imperial College Press.

11. Hinman R.S., McCrory P., Pirotta M., Relf I., Forbes A., Rossley K. M., Williamson E., Kyriakides M., Novy K., Metcalf B.R., Harris A., Reddy P., Conaghan P. G. and K.L. Bennell (2014). Acupuncture for chronic knee pain: a randomized clinical trial. JAMA, 312, (13), (pp.1313-1322),

https://www.ncbi.nlm.nih.gov/pmc/articles/PMC7439087/?fbclid=Iw AR1RNCHK3Bbdt1406sO1pJZ0Z9iPKJEbBchZ44APpHp9JcsEpx3z KE0Zv8Y

12. Huang Z., Lyu Z., Hou Z., Wu Y., Huang J., Liu F. and J. Chen (2020). Quantifying Liver-Stomach Disharmony Pattern of Functional Dyspepsia Using Multidimensional Analysis Methods. EvidenceBased Complementary and Alternative Medicine, https://downloads.hindawi.com/journals/ecam/2020/2562080.pdf

13. Keji C. and X. Hao (2003). The integration of traditional Chinese medicine and Western medicine. European Review, 11 (2), (pp. 225235).

14. Lam W.C., Lyn A. and Z. Bian (2019). Impact on Traditional Chinese Medicine and World Healthcare Systems. Pharm Med, 33, (pp. 373377).

15. Linde K., Allais G., Brinkhaus B., Fei Y., Mehring M., Shin B.C., Vickers A. and A.R. White (2016). Acupuncture for tension-type headache. Cochrane Database Syst. Rev., https://pubmed.ncbi.nlm.nih.gov/27092807/

16. Lloyd G.E.R. and J.J. Zhao (eds) (2018). Ancient Greece and China Compared. Cambridge, Cambridge University Press.

17. Lu A.P., Jia H.W., Xiao C. and Q.P. Lu (2004), Theory of traditional Chinese medicine and therapeutic method of diseases. World $J$ Gastroenterol, 10 (13), (pp. 1854-1956).

18. Maciocia G. (2015). The Foundations of Chinese Medicine. A Comprehensive Text. Amsterdam, Elsevier.

19. Manheimer E., Cheng K., Linde K., Lao L., Yoo J., Wieland S., van der Windt DAWN, Berman B.M. and L.M. Bouter (2010). Acupuncture for peripheral joint osteoarthritis (2010). 
Cochrane Database Syst. Rev.

https://pubmed.ncbi.nlm.nih.gov/20091527/

20. Merriam-Webster, merriam-webster.com

21. Qu L. (2018). Structure and distribution of the San Jiao and Cou Li Recognized interstitium in human tissues. Chinese Medicine and Culture, 1 (2), (pp. 84-87).

22. Vickers A. J. and K. Linde (2014). Acupuncture for chronic pain. JAMA, 311 (9), (pp. 955- 956).

23. Wiseman N. (2001). Translation of Chinese medical terms: not just a matter of words. Clinical Acupuncture and Oriental Medicine, 2 (1), (pp. 50-59).

24. Wiseman N. and A. Ellis (1996). Fundamentals of Chinese Medicine: Zhong Yi Xue Ji Chu. Brookline, Massachusetts, Paradigm Publications.

25. Wiseman N. and F. Ye (1998). A Practical Dictionary of Chinese Medicine. Brookline, Massachusetts, Paradigm Publications.

26. Witt C.M., Jena S., Brinkhaus B., Liecker B., Wegscheider K. and S.N. Willich (2006). Acupuncture for patients with chronic neck pain. Pain, 125 (1-2), (pp. 98-106).

27. World Federation of Chinese Medicine Societies (2008). International Standard Chinese-English Basic Nomenclature of Chinese Medicine. Beijing, China, People's Medical Publishing House.

28. World Health Organization (2019), International Classification of Diseases (ICD-11), https://icd.who.int/en

29. Xu J. and Z. Xia (2019). Traditional Chinese Medicine (TCM) - Does its contemporary business booming and globalization really reconfirm its medical efficacy \& safety? Medicine in Drug Discovery 1, https://www.sciencedirect.com/science/article/pii/S25900986119300 $03 \mathrm{X}$

30. You F., Ruan L., Zeng L. and Y. Zhang (2020). Efficacy and safety of acupuncture for the treatment of oligoasthenozoospermia: A systematic review. Andrologia, 52 (1), https://pubmed.ncbi.nlm.nih.gov/31670423/

31. Yuan J., Purepong N., Kerr D.P., Park J., Bradbury I. and S. McDonough (2008). Effectiveness of acupuncture for low back pain: a systematic review. Spine, 33 (23), (pp. 887-900).

32. Zhang G., Xiong N., Zhang Z., Liu L., Huang J., Yang J., Wu J., Lin Z. and T. Wang (2015). Effectiveness of Traditional Chinese Medicine as an Adjunct Therapy for Parkinson's Disease: A Systematic Review and Meta-Analysis. PLoS ONE, 10 (3), https://journals.plos.org/plosone/article?id=10.1371/journal.pone.011 8498 
33. Zhao Z., Li Y., Zhou L., Zhou X., Xie B., Zhang W. And J. Sun (2021). Prevention and treatment of COVID-19 using Traditional Chinese Medicine: A review. Phytomedicine, https://www.ncbi.nlm.nih.gov/pmc/articles/PMC7439087/

34. Zhou X., Li C.G., Chang D. and A. Bensoussan (2019). Current Status and Major Challenges to the Safety and Efficacy Presented by Chinese Herbal Medicine. Medicines, 6,(14), https://www.mdpi.com/23056320/6/1/14\#cite 